\title{
Oceanografia socioambiental da pesca artesanal no estado do Espírito Santo: uma análise bibliométrica
}

Este artigo tem como objetivo analisar a produção científica acadêmica sobre a atividade pesqueira artesanal no estado do Espírito Santo (ES), sudeste do Brasil com foco na Oceanografia Socioambiental a partir da abordagem bibliométrica. O levantamento bibliográfico foi realizado a partir das bases de dados 'Biblioteca Digital Brasileira de Teses e Dissertações (BDTD)', 'ISI Web of Science', 'Scirus', 'Google Scholar', 'Scielo', 'Science Direct', 'Scopus', e pelas plataformas 'Lattes' (vinculada ao Conselho Nacional de Desenvolvimento Científico e Tecnológico - CNPq) e 'Researchgate' de pesquisas realizadas no território do ES entre 1998 e 2018. Neste estudo foram identificados 48 trabalhos, sendo apenas um em universidade fora do Brasil. Dentre esses, 24 são artigos científicos (50\%) e 24 (50\%) são trabalhos acadêmicos, como monografias, dissertações e teses. 0 estudo bibliométrico permite afirmar que houve um crescimento na produção científica envolvendo a Oceanografia Socioambiental e a pesca a partir de 2006. Este aumento pode estar vinculado à implantação de cursos de pós-graduação com a temática socioambiental no ES. Os estudos encontrados envolvem conflitos socioambientais e empreendimentos ao longo da costa do ES. Pesquisas envolvendo a Oceanografia Socioambiental e pesca artesanal podem identificar conflitos e auxiliar na mitigação destes a partir do desenvolvimento do diálogo entre comunidades atingidas, órgãos gestores públicos e empreendimentos privados. Desta forma, há o incentivo à implementação da co-gestão de recursos.

Palavras-chave: Conflitos Ambientais; Gestão Costeira; Políticas Públicas; Bibliometria.

\section{Socio-environmental oceanography of artisanal fisheries in the state of Espírito Santo: a bibliometric analysis}

\begin{abstract}
This article analyzes the academic scientific production on artisanal fishing activity in the state of Espírito Santo (ES), southeast of Brazil focusing on Socioenvironmental Oceanography from the bibliometric approach. The bibliographic survey was carried out from the databases 'Brazilian Digital Library of Theses and Dissertations (BDTD)', ISI Web of Science', 'Scirus', 'Google Scholar', 'Scielo', 'Science Direct', 'Scopus', and the platforms 'Lattes' (linked to the National Council for Scientific and Technological Development - CNPq) and 'Researchgate' on research carried out in the territory of the ES between 1998 and 2018 . In this study, 48 works were identified, only one in university out of Brazil. Out of these, $24(50 \%)$ are scientific articles and $24(50 \%)$ are academic studies such as monographs, dissertations and theses. The bibliometric study allows to affirm that there was a growth in the scientific production involving the Socio-environmental Oceanography and fishing from 2006. Such increase can be linked to the implantation of postgraduate courses with the social-environmental theme in the ES. The studies found address socioenvironmental conflicts and ventures along the ES coast. Surveys involving Socio-environmental Oceanography and artisanal fishing can identify conflicts and help to mitigate them through the dialogue among affected communities, public management agencies and private enterprises. In this way, the implement co-management of resources is encouraged.
\end{abstract}

Keywords: Socioenvironmental Impacts; Coastal management; Public policy; Bibliometry.

Topic: Desenvolvimento, Sustentabilidade e Meio Ambiente

Reviewed anonymously in the process of blind peer.
Received: 05/10/2020

Approved: $26 / 11 / 2020$
Joelson Musiello Fernandes (iD)

Universidade Federal do Espírito Santo, Brasil

http://lattes.cnpq.br/8020738362355949

http://orcid.org/0000-0001-6609-4474

joelson.pesca@gmail.com

Camilah Antunes Zappes

Universidade Federal Fluminense, Brasil

http://lattes.cnpq.br/0217232489124641

http://orcid.org/0000-0002-5486-6577

camilahzappes@id.uff.br
Referencing this:

FERNANDES, J. M.; ZAPPES, C. A.. Oceanografia socioambiental da pesca artesanal no estado do Espírito Santo: uma análise bibliométrica. Revista Ibero Americana de Ciências Ambientais, v.11, n.6, p.545-558, 2020. DOI: http://doi.org/10.6008/CBPC2179$\underline{6858.2020 .006 .0044}$ 


\section{INTRODUÇÃO}

Oceanografia Socioambiental é considerada o quinto campo das subáreas tradicionais da oceanografia 'clássica', juntamente com as áreas biológica, geológica, física e química (MOURA, 2017). Na América Latina, esse ramo é denominado por 'Oceanografia Social' (e.g: social oceanography [JACQUES, 2010]) que congrega outras vertentes da ciência, com a multi, inter e transdisciplinaridade. Desta forma, insere a oceanografia clássica em linhas de pesquisa que atuam na sustentabilidade dos oceanos e co-gestão dos recursos junto à stakeholders (LUBCHENCO, 1998, MOURA, 2017; NARCHI et al., 2019).

Ainda, a Oceanografia Socioambiental estuda os oceanos a partir da interação com seres humanos por meio de métodos das Ciências Humanas e Sociais (JACQUES, 2010). Como está inserida numa esfera social/humana, essa nova área também contempla a temática da ecologia política, relacionando principalmente os conflitos socioambientais, e os direitos e deveres do Estado nos ambientes de interface terra e mar (STONIC et al., 2007; MOURA 2017).

Neste sentido, os estudos relacionados à dimensão humana se tornam uma importante ferramenta para incrementar sistemas modernos de manejo dos oceanos (CURTIN et al., 2010). Neste modelo de gestão moderno é possível estabelecer uma estrutura ecossistêmica, com objetivo principal de fornecer ferramentas para a elaboração de políticas públicas eficazes, a partir do uso racional dos recursos, mitigação de conflitos sociais, e promoção de sustentabilidade para áreas costeiras (ARBO et al., 2016).

No sudeste do Brasil, especificamente o estado do Espírito Santo (ES) possui parte de seu território constituído de zona costeira, com aproximadamente $411 \mathrm{~km}$ de linha de costa (MARTINS et al., 2011). A faixa litorânea deste estado tem sido de intenso interesse para a instalação de grandes empreendimentos desde o final do século XIX (RIBEIRO et al., 2012), e com isso, alterações socioambientais são evidenciadas com bruscas transformações econômicas, socioculturais e ambientais ao longo deste litoral (KNOX et al., 2015; MARTINS et al., 2015).

Dentre as atividades econômicas no litoral do ES, destacam-se as indústrias de energia de óleo e gás, portuária, siderúrgicas e especulação imobiliária por parte do setor turístico e residencial (KNOX et al., 2015; MUSIELLO-FERNANDES et al., 2018). Além das estruturas já implantadas, existem em análise pelo órgão estadual ambiental (Instituto Estadual de Meio Ambiente/IEMA) aproximadamente 20 estruturas portuárias em fase de consulta para implantação (KNOX et al., 2015, PINHEIRO et al., 2019a). Ao mesmo tempo em que tais empreendimentos tentam se instalar no estado, a mesma iniciativa não é verificada na criação de Unidades de Conservação Marinha (UCM). Apenas em 2018 foi criada a primeira UCM no ES sendo definida como Área de Proteção Ambiental do Arquipélago de Trindade e Martim Vaz (Decreto - 9312/2018- Ibama).

Ao longo da costa brasileira inclusive no litoral do Espírito Santo, a modernização e desenvolvimento industrial com interesses político-econômicos tem induzido intensas transformações econômicas, culturais e ambientais nas comunidades pesqueiras tradicionais (CASTRO et al., 2012; OLIVEIRA et al., 2016; SARTORE et al., 2019). Por outro lado, a gestão adequada dessas regiões é uma tarefa complexa, já que as áreas são utilizadas por com diversos usuários, em diferentes níveis, além da enorme pressão política e social (MCLEOD 
et al., 2009; SANTOS et al., 2012). Tais comunidades são as primeiras a sentir alterações nos ecossistemas costeiros, pois sua atividade econômica é realizada com embarcações de baixa autonomia atuando em áreas costeiras próximas ao seu território (CLAUZET et al., 2005; ZAPPES et al., 2016). Não obstante, essa pescaria no Brasil é marcada por conflitos e historicamente tem sido menos estudada do que a pesca industrial, e consequentemente poucos instrumentos de gestão eficazes foram desenvolvidos na resolução de problemas (LEITE et al., 2013; ANDRADE et al., 2015; RAMIRES et al., 2015).

A elaboração de políticas públicas para a pesca artesanal deve conter as características dos usuários, suas particularidades, ameaças e impactos (GRILLI et al., 2017). Por outro lado, a produção científica sobre a pesca artesanal no Brasil é incipiente, dada sua extensão e particularidades regionais. Neste sentido, este trabalho tem como objetivo realizar uma análise sistêmica e bibliométrica das informações disponíveis na literatura a partir da abordagem da Oceanografia Socioambiental sobre a pesca artesanal ao longo da costa do estado do ES.

\section{METODOLOGIA}

Para análise dos trabalhos relacionados à Oceanografia Socioambiental e pesca artesanal realizados nos limites geográficos da costa do estado do Espírito Santo foi utilizado como método a análise bibliométrica. Tal análise apresenta uma técnica quantitativa de medição que possibilita o uso de métodos estatísticos e matemáticos para mapear informações sobre determinado tema a partir de registros bibliográficos armazenados em bases de dados (ROSTAING, 1997). Neste sentido, uma pesquisa exploratória foi realizada com objetivo de descrever ou caracterizar a natureza das variáveis encontradas nas pesquisas realizadas com o tema da pesca artesanal no ES, e sua natureza, causas, relações e período.

A temática para a pesquisa bibliométrica foi 'pesca artesanal no Espírito Santo'. As palavras chaves foram formuladas para atingir o maior número de trabalhos relacionados ao tema entre os anos de 1998 e 2018 e a busca envolveu qualquer pesquisa que tivesse relação com a atividade pesqueira artesanal nos limites geográficos do litoral do ES (Figura 1). Desta forma, as palavras chaves utilizadas foram: 'pescador artesanal', 'pesca de pequena escala', 'atividade pesqueira artesanal', 'Espírito Santo'. Estas foram dispostas no banco Digital de Teses e Dissertações (BDTD) do Instituto Brasileiro de Informação Científica e Tecnológica (IBICT), Banco de Teses da CAPES, nas bibliotecas digitas de Teses e Dissertações das Universidades, ISI Web of Science, Scielo, Scirus, Google Scholar, Science Direct, Scopus, Researchgate e Lattes (a partir do nome de pesquisadores que tradicionalmente atuam em pesquisas na área e que eram encontrados nas publicações). Ainda, a análise bibliométrica foi realizada nos trabalhos encontrados para compreender a tipologia das produções [subdividida em Trabalhos de Conclusão de Cursos (TCC), Dissertações de mestrado, Teses de doutorado, Livros, Capítulos de livros e Artigos científicos], ano de publicação, as regiões do estado com maiores produções e principais temas abordados. 


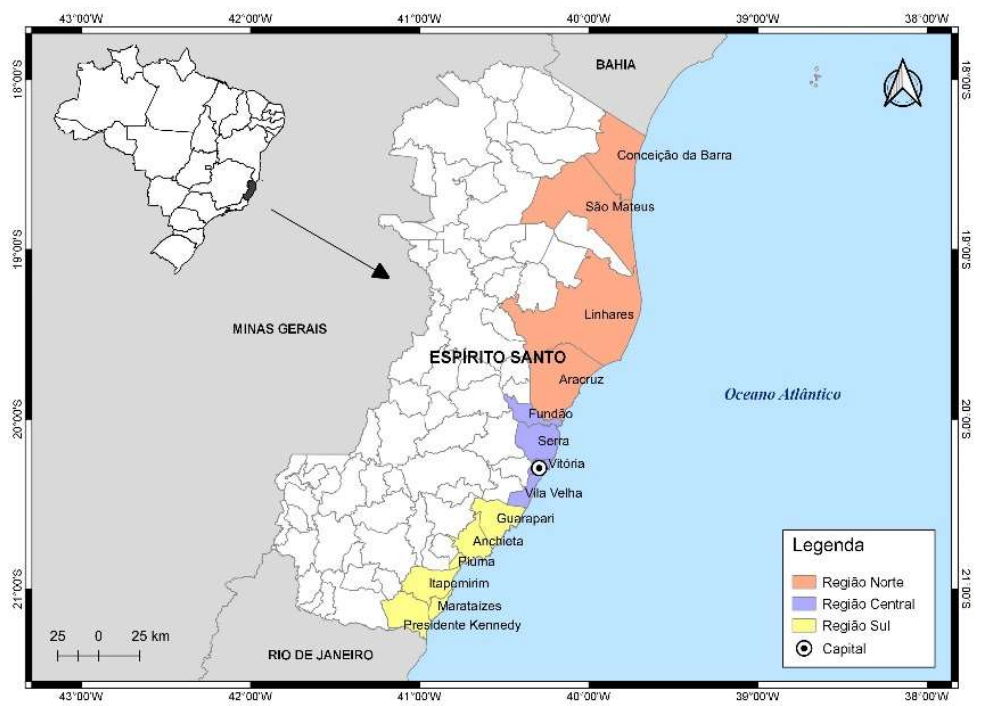

Figura 1: Municípios costeiros do estado do Espírito Santo, sudeste do Brasil, divididos por regiões.

\section{RESULTADOS}

Um total de 48 trabalhos envolvendo a área da Oceanografia socioambiental com pescadores artesanais na costa do Espírito Santo foi encontrado, sendo 24 (50\%) artigos científicos e os demais distribuídos entre dissertações de mestrado $(n=11 ; 23 \%)$, trabalhos de conclusão de curso $(n=7 ; 15 \%)$, capítulo de livro ( $n=4 ; 08 \%)$, livro $(n=1 ; 2 \%)$ e tese de doutorado $(n=1 ; 2 \%)$ (Tabela 1$)$.

Tabela 1: Lista dos títulos, ano de publicação, autores, tipologia e região geográfica dos trabalhos listados para análise bibliométrica do tema da atividade de pesca artesanal no estado do Espírito Santo, Brasil.

\begin{tabular}{|c|c|c|c|c|}
\hline TíTULO & ANO & AUTORES & TIPOLOGIA & $\begin{array}{l}\text { REGIÃO } \\
\text { GEOGRÁFICA } \\
\text { NO ES }\end{array}$ \\
\hline $\begin{array}{l}\text { Argonautas do Mangue: uma etnografia visual } \\
\text { dos caranguejeiros dos municípios da Grande } \\
\text { Vitória. }\end{array}$ & 1998 & NUNES, A. G. & Dissertação & $\begin{array}{l}\text { Região } \\
\text { Central }\end{array}$ \\
\hline $\begin{array}{l}\text { As técnicas de pesca e o conhecimento } \\
\text { tradicional envolvido nas atividades dos } \\
\text { pescadores artesanais da comunidade de Santa } \\
\text { Cruz, ES - Brasil. }\end{array}$ & 2002 & $\begin{array}{l}\text { FREITAS NETTO, R. N.; NUNES, A. G. A.; } \\
\text { ALBINO, J. }\end{array}$ & Artigo & Região Norte \\
\hline $\begin{array}{l}\text { A pesca realizada na comunidade de } \\
\text { pescadores artesanais de Santa Cruz/ES-Brasil }\end{array}$ & 2002 & $\begin{array}{l}\text { FREITAS NETTO, R. N.; NUNES, A. G. } \\
\text { A.;ALBINO, J. }\end{array}$ & Artigo & Região Norte \\
\hline $\begin{array}{l}\text { Avaliação multidimensional dos sistemas } \\
\text { pesqueiros da região sul do Espírito Santo, } \\
\text { Brasil, e seus indicadores de sustentabilidade. }\end{array}$ & 2004 & PIZETTA, G. T. & TCC & Todo estado \\
\hline $\begin{array}{l}\text { Descrição dos impactos sofridos pela } \\
\text { comunidade de pescadores artesanais de Santa } \\
\text { Cruz - ES, Brasil }\end{array}$ & 2004 & FREITAS NETTO, R. & Artigo & Região Norte \\
\hline $\begin{array}{l}\text { Impacto da Mitilicultura no desenvolvimento } \\
\text { das comunidades tradicionais ao entorno das } \\
\text { Praias da Cerca e Guaibura, Guarapari, ES. }\end{array}$ & 2005 & OLIVEIRA, B. L. & TCC & Região Sul \\
\hline Dinâmica da frota linheira de Itaipava, ES. & 2006 & STEIN, C. E. & TCC & Região Sul \\
\hline $\begin{array}{l}\text { A pesca do camarão em Conceição da Barra, ES, } \\
\text { como um estudo multidisciplinar do colapso de } \\
\text { um sistema pesqueiro. }\end{array}$ & 2006 & RABELO, L. B. & TCC & Região Norte \\
\hline $\begin{array}{l}\text { Caracterização e avaliação da atividade de } \\
\text { pesca amadora na Praia de Camburi, Vitória - } \\
\text { ES. }\end{array}$ & 2006 & CHIAPPANI, L. H. B. & TCC & $\begin{array}{l}\text { Região } \\
\text { Central }\end{array}$ \\
\hline $\begin{array}{l}\text { Avaliação bio-ecológica, tecnológica e } \\
\text { socioeconômica do sistema pesqueiro de } \\
\text { Itapoã, Vila Velha-ES. }\end{array}$ & 2006 & ARAÚJO, M. F. & TCC & $\begin{array}{l}\text { Região } \\
\text { Central }\end{array}$ \\
\hline $\begin{array}{l}\text { Diversidade de artefatos de pesca artesanal } \\
\text { marinha do Espírito Santo. }\end{array}$ & 2007 & $\begin{array}{l}\text { FREITAS NETTO, R.; DI BENEDITTO, A. P. } \\
\text { M. }\end{array}$ & Artigo & Todo estado \\
\hline
\end{tabular}


Contribuição ao conhecimento da distribuição da toninha Pontoporia blainvillei (Gervais \& d’Orbigny, 1844) no estado do Espírito Santo, sudeste do Brasil.

Interactions between fisheries and cetaceans in Espírito Santo coast, Southeastern Brazil.

Entre barracões e módulos de pesca: pescaria e meio ambiente na regulação do uso de espaços públicos na Barra do Jucu (Vila Velha- ES).

Profile of social actors as a tool the definition of marine protected areas: the case of the llha dos Franceses, southern coast of Espírito Santo, Brazil.

Produção pesqueira no triênio 2003-2005 pela cooperativa de pesca de Vila Velha, Espírito Santo, sudeste do Brasil.

Poluição do ar atmosférico e doenças respiratórias em pescadores de Anchieta/ES.

O conhecimento ecológico tradicional como ferramenta para mapeamento de ambientes marinhos.

Uma análise da percepção ambiental e transformação socioeconômica de uma comunidade de pescadores artesanais em região estuarina no sudeste do Brasil.

Potencialidade social e econômica da pesca e maricultura no estado do Espírito Santo, Brasil. Mapeando e valorizando o conhecimento ecológico tradicional na gestão de áreas marinhas protegidas.

Traditional Ecological Knowledge and the mapping of benthic marine habitats.

A pesca de camarão em Conceição da Barra, Espírito Santo, como um estudo multidisciplinar do colapso de um sistema pesqueiro.

A rápida expansão recente da pesca de Itaipava, suas causas e consequências: um estudo de caso.

Entre o desenvolvimento econômico e a preservação ambiental: O Caso da Pesca Artesanal e a Configuração de um Cenário de Injustiça Ambiental em Regência Augusta-ES.

Movimentos das Águas Caboclas: narrativa visual, cotidiano e ruptura na comunidade pesqueira de Regência Augusta-ES.

Os impactos socioambientais do desenvolvimento econômico na pesca artesanal: uma análise a partir dos modos de vida e de trabalho.

\begin{tabular}{|c|c|c|c|c|}
\hline $\begin{array}{l}\text { A pesca artesanal, conflitos e novas } \\
\text { configurações. }\end{array}$ & 2014 & KNOX, W.; TRIGUEIRO, A. & Artigo & Todo estado \\
\hline $\begin{array}{l}\text { Produção da cultura e mediação social do } \\
\text { ambiente nas pescas artesanais das } \\
\text { comunidades vizinhas de Ubú e Parati no } \\
\text { Espírito Santo }\end{array}$ & 2014 & PEREIRA, G. R. & Dissertação & Região Sul \\
\hline A Pesca Artesanal no Litoral do ES & 2015 & KNOX, W.; TRIGUEIRO, A. & Livro & Todo estado \\
\hline $\begin{array}{l}\text { Aspectos do fenômeno jurídico entre os } \\
\text { pescadores da Barra do Jucu, Vila Velha-ES. }\end{array}$ & 2015 & FILGUEIRAS, M. P. & $\begin{array}{l}\text { Capítulo de } \\
\text { Livro }\end{array}$ & Região Sul \\
\hline $\begin{array}{l}\text { "A turma aqui gosta de } \\
\text { representações sobre a pesca } \\
\text { amadora }\end{array}$ & 2015 & GUIMARÃESM R. A.; FANTINEL, F. D. & Artigo & $\begin{array}{l}\text { Região } \\
\text { Central }\end{array}$ \\
\hline
\end{tabular}

\section{7 \\ FREITAS NETTO, R.; SICILIANO, S. \\ Artigo \\ Todo estado}

\begin{tabular}{llll}
2008 & FREITAS NETTO, R; DI BENEDITTO, A. P. & Artigo & Todo estado \\
& M. & & \\
\hline 2008 & FILGUEIRAS, M. P. & Dissertação & Região Sul \\
\hline
\end{tabular}

2009 PINHEIRO, H. T.; FERREIRA, A. L.; Artigo

Região Sul MOLINA, R. P.; PROTTI, L. M. C.; ZANARDO, S. C.; JOYEUX, J. C.; DOXSEY, J. R.

2009 FREITAS NETTO; R.; KROHLING, W.; Artigo Região ROCHA, M. B.; DI BENEDITTO, A. P. M. Central

\begin{tabular}{llll}
2011 & DORNELLAS, F. S. D. & Dissertação & Região Sul \\
\hline 2011 & TEIXEIRA, J. B. & Dissertação & Região Sul \\
\hline 2012 & ROCHA, K. S.; SILVA, R. V.; RANDOW, R. & Artigo & Região Norte \\
2012 & $\begin{array}{l}\text { TEIXEIRA, J. B.; LIMA, A. C.; BOECHAT, F. } \\
\text { B.; RODRIGUES, R. L. }\end{array}$ & Artigo & Todo estado \\
2013 & $\begin{array}{l}\text { TAVARES, M. N. } \\
\end{array}$ & Dissertação & Região Norte
\end{tabular}

2013 TEIXEIRA, J. B.; MARTINS, A. S.; Artigo Região Sul PINHEIRO, H. T.; SECCHIN, N. A.; MOURA, R. L.; BASTOS, A. C.

2014 MARTINS, A. S.; RABELO, L. B.; DOXSEY, Capítulo de Região Norte J. R.; SOUSA, C. R.; FANCHIOTTI- LivrO MEIRELES, A.; RODRIGUES, C. M.; PIZETTA, G. T.; ARAÚJO, J. S.; SANTOS, L. B.; ZAMBON, M. C.; SILVA, M. P. C.

2014 MARTINS, A. S.; SANTOS, L. B.; SILVA, Capítulo de Região Sul M. P. C.; DOXSEY, J. R.; SOUSA, C. R.; Livro MEIRELES, A. F.; RODRIGUES, C. M.; PIZETTA, G. T.; ARAÚJO, J. S.; ZAMBON, M. C.; RABELO, L. B.

2014 MERÇON, F. A. M. Dissertação $\quad$ Região Norte

2014 BICALHO, C.; TRIGUEIRO, A.; KNOX, W.; Artigo Região Norte BEHR, R.

2014 WINIFRED, K.; TRIGUEIRO, A.; ZANETTI, Artigo Região

D. Central 
embarcada no late Clube do Espírito Santo.

Avaliação da atividade pesqueira numa comunidade de pescadores artesanais no Espírito Santo, Brasil.

Competition for space between fishing and exploratory oil drilling, observed from a drilling platform in the Espírito Santo basin, southeastern Brazil.

\begin{tabular}{|c|c|c|c|c|}
\hline $\begin{array}{l}\text { A relação saúde-doença entre mulheres } \\
\text { pescadoras artesanais de São Mateus, ES. }\end{array}$ & 2015 & MARTINS, Q. S. & Dissertação & Região Norte \\
\hline $\begin{array}{l}\text { Sustentabilidade das atividades pesqueiras do } \\
\text { município de Piúma, litoral sul do Espírito } \\
\text { Santo, Brasil }\end{array}$ & 2015 & $\begin{array}{l}\text { BASILIO, T. H.; SILVA, E. V.; FIORESI, D. } \\
\text { B.; GOMES, M. P.; GARCEZ, D. S. }\end{array}$ & Artigo & Região Sul \\
\hline $\begin{array}{l}\text { Pescadores artesanais e comunidade } \\
\text { tradicional da ilha das Caieiras/Vitória em } \\
\text { perspectiva histórica }\end{array}$ & 2016 & CORREA, F. R.; VASCONCELOS, F. N. & $\begin{array}{l}\text { Capítulo de } \\
\text { Livro }\end{array}$ & $\begin{array}{l}\text { Região } \\
\text { Central }\end{array}$ \\
\hline $\begin{array}{l}\text { Análise integrada de unidades geoecológicas } \\
\text { relacionadas com as atividades pesqueiras no } \\
\text { litoral sul do Espírito Santo, Brasil. }\end{array}$ & 2016 & $\begin{array}{l}\text { T. H. BASILIO; D. S. GARCEZ; C. N. } \\
\text { BODART; E. V. SILVA }\end{array}$ & Artigo & Região Sul \\
\hline $\begin{array}{l}\text { Mulheres na atividade pesqueira no Espírito } \\
\text { Santo }\end{array}$ & 2016 & KNOX, W.; FIRME, R. M. & Artigo & $\begin{array}{l}\text { Região } \\
\text { Central }\end{array}$ \\
\hline $\begin{array}{l}\text { Pesca artesanal e industrialização na Barra do } \\
\text { Riacho, Aracruz - ES. }\end{array}$ & 2016 & LYRIO, I. J. P. L. & Dissertação & Região Norte \\
\hline $\begin{array}{l}\text { Manguezais sob uma perspectiva social e } \\
\text { econômica: percepção ambiental dos serviços } \\
\text { ecossistêmicos presentes na ilha das caieiras, } \\
\text { Vitória, ES. }\end{array}$ & 2017 & TIENGO, R. D.P. & TCC & $\begin{array}{l}\text { Região } \\
\text { Central }\end{array}$ \\
\hline $\begin{array}{l}\text { Small-scale shrimp fisheries on the Brazilian } \\
\text { coast: Stakeholders perceptions of the closed } \\
\text { season and integrated management. }\end{array}$ & 2017 & $\begin{array}{l}\text { MUSIELLO-FERNANDES, J.; ZAPPES, C. } \\
\text { A.; HOSTIM-SILVA, M. }\end{array}$ & Artigo & Todo estado \\
\hline $\begin{array}{l}\text { Aspectos etnobiológicos e etnoecológicos na } \\
\text { pesca artesanal em três municípios do litoral } \\
\text { sul do Espírito Santo. }\end{array}$ & 2018 & OLIVEIRA, A. C. M. & Dissertação & Região Sul \\
\hline $\begin{array}{l}\text { Pesca camaroneira e conhecimento ecológico } \\
\text { local dos pescadores artesanais de camarão na } \\
\text { costa central do Brasil: implicações } \\
\text { conservacionistas. }\end{array}$ & 2018 & MUSIELLO-FERNANDES, J. & Tese & Todo estado \\
\hline $\begin{array}{l}\text { Small-scale fisheries of the Atlantic seabob } \\
\text { shrimp (Xiphopenaeus kroyeri): Continuity of } \\
\text { commercialization and maintenance of the } \\
\text { local culture through making public policies on } \\
\text { the Brazilian coast. }\end{array}$ & 2018 & $\begin{array}{l}\text { MUSIELLO FERNANDES, J.; ZAPPES, C.A. } \\
\text { HOSTIM-SILVA, M. }\end{array}$ & Artigo & Todo estado \\
\hline $\begin{array}{l}\text { Pesca artesanal e as interferências sobre a } \\
\text { atividade na mesorregião central do Espírito } \\
\text { Santo. }\end{array}$ & 2018 & $\begin{array}{l}\text { MUSIELLO-FERNANDES, J.; VIEIRA, F. } \\
\text { V.; FLORES, R. M.; CABRAL, L.; ZAPPES, } \\
\text { C. A. }\end{array}$ & Artigo & Região Sul \\
\hline $\begin{array}{l}\text { Índice de potencialidade socioeconômica, } \\
\text { produtiva e ambiental de comunidades } \\
\text { pesqueiras situadas no norte do estado do } \\
\text { Espírito Santo (IPSPA - norte). }\end{array}$ & 2018 & GOMES, V. A. P. G.; FREITAS, R. R. & Artigo & Região Norte \\
\hline $\begin{array}{l}\text { "Zonas de Sacrifício": } \quad \text { Impactos } \\
\text { socioambientais e a pesca artesanal em Barra } \\
\text { do Riacho, Aracruz (ES). }\end{array}$ & 2018 & VIEIRA, L. H. & Artigo & Região Norte \\
\hline
\end{tabular}

2015 MARTINS, N. G.; RODRIGUES, D. A.; Artigo Região Norte RIBEIRO, G. M.; FREITAS, R. R.

2015 SILVA, A. C. T.; VALENTIN, J. L.; Artigo Região Norte VIANNA, $M$.

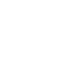

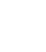
. 
= 09; 21\%), sendo as regiões norte e sul com maior parcela de estudos, com 15 (31\%) e 14 estudos, respectivamente. Os estudos realizados em mais de uma região, ou em todo estado simultaneamente totalizaram 10, representando $21 \%$ do total (Figura 3).

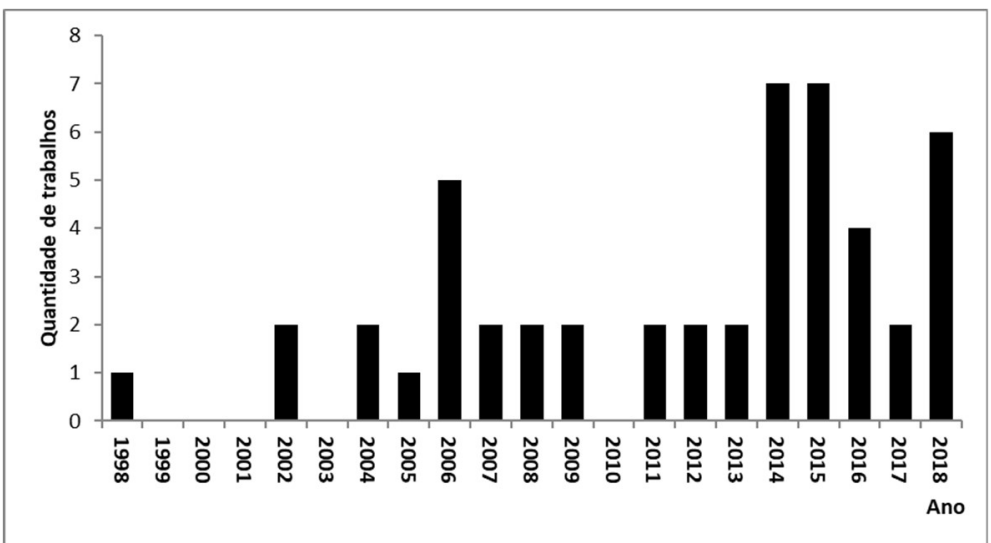

Figura 2: Distribuição temporal das pesquisas envolvendo Oceanografia Socioambiental e pesca artesanal realizadas ao longo da costa do estado do Espírito Santo, sudeste do Brasil.

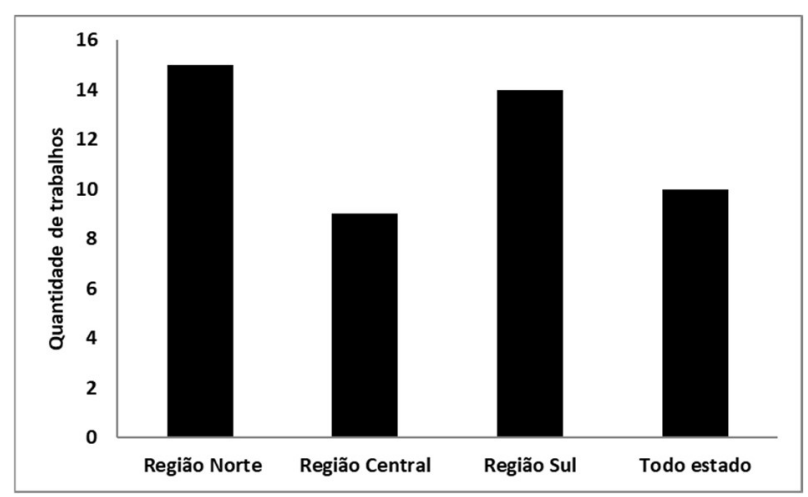

Figura 3: Distribuição espacial das pesquisas envolvendo Oceanografia Socioambiental e pesca artesanal realizadas ao longo da costa do estado do Espírito Santo, sudeste do Brasil.

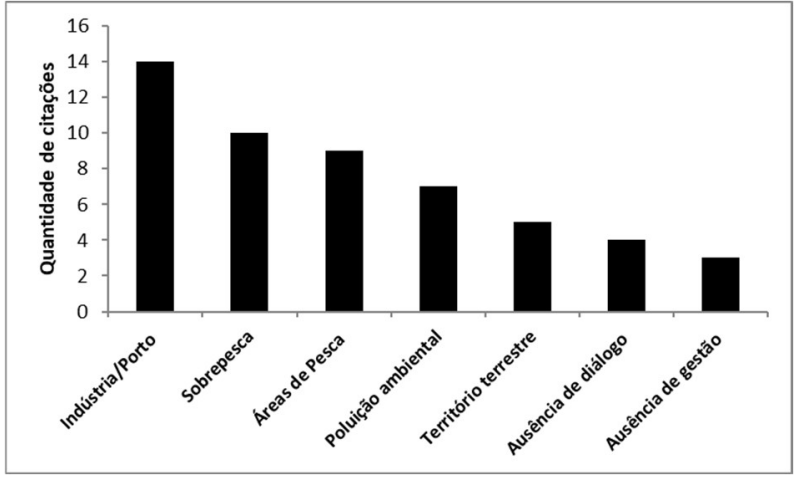

Figura 4: Temática das pesquisas envolvendo Oceanografia Socioambiental e pesca artesanal realizada ao longo da costa do estado do Espírito Santo, sudeste do Brasil.

Os trabalhos acadêmicos (TCC's dissertações de mestrado e tese de doutorado) foram realizados em diferentes áreas do conhecimento. A Universidade Federal do Espírito Santo (UFES) foi a instituição de ensino e pesquisa que mais publicou sobre o tema em que o Departamento de Oceanografia e Ecologia da UFES apresentou as maiores contribuições $(n=10)$, seguido das áreas de Ciências Sociais $(n=4)$, Geografia $(n=2)$ e Medicina $(n=2)$ e Antropologia $(n=1)$.

Em relação aos temas abordados nos trabalhos analisados, 34 (71\%) pesquisas abordam como tópico principal do estudo a pesca artesanal em decorrência de algum conflito ou problema socioambiental. Dentre as intervenções negativas descritas, a mais frequente está relacionada à interferência de indústria ou portos sobre a pesca artesanal ( $n=14 ; 27 \%$ ), sendo os ramos econômicos mais citados 1 ) exploração de óleo e gás; 2) mineração; e 3) Celulose. Os portos estão associados ao escoamento dos produtos destas indústrias. Outros problemas encontrados nos trabalhos envolvem: sobrepesca $(n=10 ; 19 \%)$, conflitos com os territórios de pesca $(n=9 ; 17 \%)$, poluição ambiental $(n=7 ; 13 \%)$, território terrestre (turismo e especulação imobiliária) ( $n=5 ; 10 \%$ ), ausência de diálogo com órgãos ambientais ( $n=4 ; 8 \%$ ) e ausência de gestão 
pesqueira $(n=3 ; 6 \%)$. Destaca-se que o número de conflitos $(n=52)$ é maior que o número de trabalhos envolvendo pesca artesanal e conflito $(n=32)$, pois uma mesma pesquisa citou mais de um conflito (Figura $4)$.

\section{DISCUSSÃO}

A produção científica é um recurso indispensável para promover o desenvolvimento da ciência e tecnologia (WEITZEL, 2006). Neste sentido, o estudo sobre a configuração de campos científicos por meio da análise bibliométrica é importante, pois fornece um diagnóstico da atividade acadêmica sobre determinado tema, elucidando sua estrutura intelectual (MOYA-ANÉGON et al., 2005), e pode servir como base para elaboração das medidas de gestão.

No estado do Espírito Santo, a maior parte das pesquisas relacionadas à Oceanografia Socioambiental e pesca artesanal está mais disponível no formato de artigos científicos publicados em revistas (periódicos) nacionais e internacionais. O artigo científico é um meio de comunicação amplamente utilizado por pesquisadores, com autoria declarada em que são discutidas ideias, métodos, técnicas e resultados em diversas áreas do conhecimento. Os periódicos cumprem o papel de divulgar o trabalho de pesquisa à comunidade científica e à sociedade como um todo (SEVERINO, 2002). Ao mesmo tempo, artigo científico é uma importante ferramenta para a organização do conhecimento na elaboração de políticas públicas voltadas para sustentabilidade (LEE, 1993; WIDMER, 2009). Os cientistas que realizam estudos na área socioambiental querem que suas pesquisas tenham um efeito prático na elaboração de políticas públicas (SINGH et al., 2014). Na prática, poucos são os estudos utilizados na formulação de políticas, gestão ou na esfera pública (YOUNG et al., 2016). Devido a isso, há uma política de incentivo na área científica para que pesquisadores atuem mais na formulação dessas políticas contribuindo com seu conhecimento (KLINGER et al., 2018).

De uma maneira geral para o estado do ES, houve um incremento na quantidade de estudos com a temática socioambiental e pesca artesanal nos últimos 20 anos. Tal incremento é relacionado à implantação dos cursos de graduação e Programas de Pós-Graduação (PPG) nas áreas correlatas, principalmente a partir do ano de 2006. A UFES é a mais antiga e principal instituição de ensino e pesquisa do estado onde a partir do ano 2000 foram implementados PPG's com a temática da socioambiental como, as áreas de Oceanografia, Ciências Biológicas e Geografia. Neste mesmo período também houve um incremento do conhecimento sobre a produção pesqueira da pesca artesanal no território do ES. Tal incremento ocorreu a partir do desenvolvimento de pesquisas realizadas principalmente por tais PPG's. Desta forma, fica evidente que a Oceanografia Socioambiental pode permear diversas áreas do conhecimento, e tem como base a interdisciplinaridade, a partir das relações humanas com o sistema costeiro/marinho, assim como apontada por diversos autores, e.g: Espinoza-Tenorio et al. (2014), Moura (2017) e Narchl et al. (2019).

Não foi observada uma grande variação da produção científica na temática socioambiental e pesca artesanal ao longo da costa capixaba. Destaca-se que a região sul do estado apresenta a maior quantidade de portos/empresas (empreendimentos) sendo 3 na região litorânea, 2 na região centro e 1 na região norte 
(PINHEIRO et al., 2019a). Por outro lado, na região norte a malha rodoviária é ineficiente e as comunidades pesqueiras estão distantes geograficamente dos centros urbanos, com uma pescaria mais costeira, quando comparado às demais regiões (PINHEIRO et al., 2007; MARTINS et al., 2013; MUSIELLO-FERNANDES et al., 2018).

A mesorregião do estado é a região com menor aporte de conhecimento sobre a pesca artesanal. Esta região é caracterizada com urbanizada, bem como é o local onde está inserida a capital do estado (Vitória), e abarcam os dois mais altos índices de IDH's (Índice de Desenvolvimento Humano Municipal) e PIB's (Produto Interno Bruto) do estado (GIRARDI et al., 2006). Ao longo da costa brasileira, os estudos em áreas urbanas são difíceis, pois os pesquisadores são confundidos com agentes de fiscalização ambiental (ALVES et al., 2010).

Além disso, a atividade de pesca em áreas urbanas é alvo de conflitos ambientais, com profundas alterações nos modos de vida dos pescadores, e consequentemente uma perda de territórios e exclusão social causada pela crescente urbanização (SILVA, 2011; SANTOS et al., 2013; PEDROSA et al., 2013). Como consequência, os problemas sociais são evidentes, como o consumo excessivo de bebidas alcoólicas, entorpecentes e as enfermidades inerentes à atividade, por conta das longas jornadas de trabalho (NASCIMENTO et al., 2007; PENA et al., 2011). Estes fatores influenciam diretamente na continuidade da atividade pesqueira urbana, e em muitos casos passa a ser atividade econômica secundária, afastando do oficio as gerações seguintes, comprometendo a transferência do conhecimento tradicional (LIMA et al., 2012; SANTOS et al., 2013).

No ES, vários estudos envolvendo Oceanografia Socioambiental e pesca artesanal discutem conflitos causados por empreendimentos sobre comunidades pesqueiras (CUNHA, 2003; OLIVEIRA et al., 2016). A literatura desta temática tende a aumentar já que no estado existem várias propostas para instalação de novos empreendimentos no litoral sobrepondo territórios de pesca artesanal (Pinheiro et al., 2019a). A região costeira/oceânica proporciona benefícios econômicos principalmente ao setor privado. A exploração em larga escala destes ecossistemas intensifica conflitos socioambientais prejudicando principalmente comunidades que dependem da pesca artesanal (LITTLE, 2001; ALLISON et al., 2012; CASTRO et al., 2012 ; STOJANOVIC et al., 2013; GASALA et al., 2016; ZAPPES et al., 2016). Estes conflitos podem ser verificados entre diferentes setores da sociedade, como a indústria de exploração de óleo e gás, o colapso dos estoques pesqueiros, o turismo descontrolado, a indústria portuária, e especulação imobiliária (KLINGER et al., 2018; MUSIELLO-FERNANDES et al., 2018; SATORE et al., 2019). Por outro lado, com o objetivo de fortalecer o crescimento econômico, o governo brasileiro alterou recentemente as regras do licenciamento ambiental favorecendo projetos de empreendimentos, como portos e mineração (PINHEIRO et al., 2019b) pouco considerando o bem-estar sociocultural. Frente a este cenário político é necessário realizar estudos envolvendo a Oceanografia Socioambiental, pesca artesanal e gestão pública do litoral do ES a fim de se manter qualidade de vida para as comunidades pesqueiras artesanais e sustentabilidade do ambiente costeiro/marinho. 


\section{CONCLUSÕES}

O conhecimento científico envolvendo pesca artesanal e questões socioambientais no estado do Espírito Santo teve um incremento, principalmente após 2006. Todavia, lacunas do conhecimento ainda precisam ser preenchidas, tendo em vista a importância socioeconômica da atividade pesqueira artesanal, os conflitos existentes e a possibilidade do aumento de empreendimentos da região costeira do estado.

Por meio da Oceanografia Socioambiental como um novo ramo das Ciências Ambientais é possível abranger diversas áreas do conhecimento, pautados na relação social com a região costeira/oceânica. Estudos com tal temática podem identificar conflitos socioambientais, e com isso, auxiliar na mitigação desses problemas a partir do diálogo entre comunidades de pesca artesanal, órgãos ambientais, instituições de ensino e pesquisa e empreendimentos costeiros. Ainda, tais estudos podem aumentar e garantir a participação de stakeholders na elaboração de estratégias de co-gestão do bom uso dos ecossistemas costeiros.

AGRADECIMENTOS: O primeiro autor agradece a Coordenação de Aperfeiçoamento de Pessoal de Nível Superior - CAPES [Processo 88882315888/2019-1] pela bolsa de estudo. A segunda autora agradece à Fundação Carlos Chagas Filho de Amparo à Pesquisa do Rio de Janeiro - FAPERJ [E-26/203.202/2016; E26/202.789/2019] e ao Conselho Nacional de Desenvolvimento Científico e Tecnológico - CNPq [Processo 400053/2016-0] pelo financiamento às pesquisas.

\section{REFERÊNCIAS}

ALLISON, E. H.; RATNER, B. D.; ASGARD, B.; WILLMANN, R. POMEROY, R.; KURIEN, J.. Rights Based Governance: from fishing rights to human rights. Fish and Fisheries, v.13, p.1429, 2012. DOI: http://doi.org/10.1111/j.14672979.2011.00405.x

ANDRADE, J. C. P.; SCHIAVETTI, A.. Artisanal fishing and local conflicts: the case of the 'Pedras de Una' fishing community, Bahia, Brazil. Revista de Gestão Costeira Integrada, v.15, n.3, p.425-438, 2015. DOI: http://doi.org/10.5894/rgci536

ARAÚJO, M. F.. Avaliação bio-ecológica, tecnológica e socioeconômica do sistema pesqueiro de Itapoã, Vila Velha/ES. Monografia (Bacharelado em Oceanografia) Universidade Federal do Espírito Santo, Vitória, 2006.

ARBO, P.; THUY, P. T. T.. Use conflicts in ecosystem-based management - The case of oil versus fisheries. Ocean and Coastal Management, v.122, p.77-86, 2016. DOI: http://doi.org/10.1016/j.ocecoaman.2016.01.008

BASILIO, T. H.; SILVA, E. V.; FIORESI, D. B.; GOMES, M. P.; GARCEZ, D. S.. Sustentabilidade das atividades pesqueiras do município de Piúma, litoral sul do Espírito Santo, Brasil. Arquivos de Ciências do Mar, v.48, n.1, p.69-86, 2015.

BASILIO, T. H.; GARCEZ, D. S.; BODART, C. N.; SILVA, E. V.. Análise integrada de unidades geoecológicas relacionadas com as atividades pesqueiras no litoral sul do Espírito Santo, Brasil. Revista de Gestão Costeira Integrada, v.16, n.2, p.163-170, 2016. DOI: http://doi.org/10.5894/rgci586

BICALHO, C.; TRIGUEIRO, A.; KNOX, W.; BEHR, R.. Movimentos das Águas Caboclas: narrativa visual, cotidiano e ruptura na comunidade pesqueira de Regência Augusta/ES. Caderno eletrônico de Ciências Sociais, v.2, n.1, p.19-42, 2014.

CASTRO, S. M.; ALMEIDA, J. R.. Dragagem e conflitos ambientais em portos clássicos e modernos: Uma revisão. Sociedade e Natureza, v.24, n.3, p. 519-534, 2012. DOI: http://doi.org/10.1590/S1982-45132012000300011

CHIAPPANI, L. H. B.. Caracterização e avaliação da atividade de pesca amadora na Praia de Camburi, Vitória - ES. Monografia (Bacharelado em Oceanografia) - Universidade Federal do Espírito Santo, Vitória, 2006.

CLAUZET, M.; RAMIRES, M.; BARRELLA, W.. Pesca artesanal e conhecimento local de duas populações Caiçaras (Enseada do Mar Virado e Barra do Una) no Litoral de São Paulo, Brasil. Multiciência, v.4, p.1-22, 2005.

CUNHA, I.. Conflito ambiental em águas costeiras: Relação porto - cidade no Canal de São Sebastião. Ambiente \& Sociedade, v.6, n.2, p.83-98, 2003.

CORREA, F. R.; VASCONCELOS, F. N.. Pescadores artesanais e comunidade tradicional da ilha das Caieiras/Vitória em perspectiva histórica. Vitória, 2016. 
CURTIN, R.; PRELLEZO, R.. Understanding marine ecosystem based management: A literature review. Marine Policy, v.34, p.821-830, 2010. DOI:

http://doi.org/10.1016/j.marpol.2010.01.003

DORNELLAS, F. S. D.. Poluição do ar atmosférico e doenças respiratórias em pescadores de Anchieta/ES.

Dissertação (Mestrado em Gestão Pública) - Escola Superior de Ciências da Santa Casa de Misericórdia de Vitória, Vitória, 2011.

ESPINOZA-TENORIO, A.; MORENO-BÁEZ, M.; PECH, D.; VILLALOBOS-ZAPATA, G. J.; VIDALHERNÁNDEZ, L.; RAMOSMIRANDA, J.; MENDOZA-CARRANZA, M.; ZEPEDADOMÍNGUEZ, J. A.. El ordenamiento ecológico marino en México: un reto y una invitación al quehacer científico. Latin American Journal of Aquatic Research, v.42, n.3, p.386-400, 2014. DOI: http://doi.org/10.3856/vol42-issue3-fulltext-1

FILGUEIRAS, M. P.. Entre barracões e módulos de pesca: pescaria e meio ambiente na regulação do uso de espaços públicos na Barra do Jucu (Vila Velha- ES). Dissertação (Mestrado) - Universidade Federal Fluminense, Fluminense, 2008.

FILGUEIRAS, M. P.. Aspectos do fenômeno jurídico entre os pescadores da Barra do Jucu, Vila Velha/ES. In: KNOX, W.; TRIGUEIRO, A.. Saberes, Narrativas e Conflitos na pesca artesanal. Vitória: EDUFES, 2015. p.161-186.

FREITAS NETTO, R.. Descrição dos impactos sofridos pela comunidade de pescadores artesanais de Santa Cruz/ES, Brasil. Bioikos, v.18, n.1, p.51-62, 2004.

FREITAS NETTO, R. N.; NUNES, A. G. A.; ALBINO, J.. As técnicas de pesca e o conhecimento tradicional envolvido nas atividades dos pescadores artesanais da comunidade de Santa Cruz, ES, Brasil. Geografares, v.3, p.123-132, 2012.

FREITAS NETTO, R. N.; NUNES, A. G. A.; ALBINO, J.. A pesca realizada na comunidade de pescadores artesanais de Santa Cruz/ ES - Brasil. Boletim do Instituto de Pesca, v. 28, n.1, p.93-100, 2002.

FREITAS NETTO, R.; BENEDITTO, A. P. M. D.. Diversidade de artefatos da pesca artesanal marinha do Espírito Santo. Biotemas, v.20, n.2, p.107-119, 2007.

FREITAS NETTO, R.; SICILIANO, S.. Contribuição ao conhecimento da distribuição da toninha Pontoporia blainvillei (Gervais \& d'Orbigny, 1844) no estado do Espírito Santo, sudeste do Brasil. Museu de Biologia Melo Leitão, v.21, p.35-45, 2007.

FREITAS NETTO, R.; KROHLING, W.; ROCHA, M. B.; DI BENEDITTO, A. P. M.. Produção pesqueira no triênio 20032005 pela cooperativa de pesca de Vila Velha, Espírito Santo, sudeste do Brasil. Boletim do Instituto de Pesca, v.35, n.4, p.663-679, 2009.

FREITAS NETTO, R.; DI BENEDITTO, A. P. M.. Interactions between fisheries and cetaceans in Espírito Santo coast, Southeastern Brazil. Zoociências, v.10, n.1, p.55-63, 2008. DOI: http://doi.org/10.34019/2596-3325.2008.v10.24086

GASALLA, M. A.; GANDINI, F. C.. The loss of fishing territories in coastal areas: the case of seabob-shrimp small-scale fisheries in São Paulo, Brazil. Maritime Studies, n.15, p.9-18, 2006. DOI: http://doi.org/10.1186/s40152-016-0044-2

GIRARDI, G.; COMETTI, R. S.. Dinâmica do uso e ocupação do solo no litoral sul do estado do Espírito Santo, Brasil. Desenvolvimento e Meio Ambiente, v.13, p.51-73, 2006. DOI: http://doi.org/10.5380/dma.v13i0.3114

GOMES, V. A. P. G.; FREITAS, R. R.. Índice de potencialidade socioeconômica, produtiva e ambiental de comunidades pesqueiras situadas no norte do estado do Espírito Santo (IPSPA - norte). Revista Produção Online, v.18, n.1, p.36-62, 2018. DOI: http://doi.org/10.14488/1676-1901.v18i1.2568

GRILLI, N. M.; XAVIER, L. Y.; JACOBI, P. R.; TURRA, A.. Sustentabilidade das regiões costeiras e oceânicas? Necessidade de um novo relacionamento entre ciência e gestão. Revista USP, v.113, p.45-58, 2017. DOI: http://doi.org/10.11606/issn.2316-9036.v0i113p45-58

GUIMARÃES, R. A.; FANTINEL, F. D.. "A turma aqui gosta de ganhar": representações sobre a pesca amadora embarcada no late Clube do Espírito Santo. Revista de Estudos Organizacionais e Sociedade, v.2, n.4, p.365-418, 2015. DOI: http://doi.org/10.25113/farol.v2i4.2537

IZOTON, I. J. P. L.. Pesca artesanal e industrialização na Barra do Riacho, Aracruz/ES. Monografia (Bacharelado) Universidade Federal do Espírito Santo, Vitória, 2016.

JACQUES, P. J.. The social oceanography of top oceanic predators and the decline of sharks: A call for a new field. Progress in Oceanography, v.86, p.192-203, 2010. DOI: http://doi.org/10.1016/j.pocean.2010.04.001

KLINGER, D.; EIKESET, B.; DAVÍGSDÓTTIR, A.; WINTER, M.; WATSON, J.. The mechanics of blue growth: management of oceanic natural resource use with multiple, interacting sectors. Marine Policy, v.87, p.356-362, 2018. DOI: http://doi.org/10.1016/j.marpol.2017.09.025

KNOX, W.; FIRME, R. M.. Mulheres na atividade pesqueira no Espírito Santo. Revista Gênero, v.16, n.2, p.219-235, 2016.

KNOX, W.; TRIGUEIRO, A.. A pesca artesanal, conflitos e novas configurações. Revista Espaço de Diálogo e Desconexão, v.8, n.1, p.1-09, 2014. DOI: http://doi.org/10.32760/1984-1736/REDD/2014.v8i2.6956

KNOX, W.; TRIGUEIRO, A.. Saberes, narrativas e conflitos na pesca artesanal. Vitória: EDUFES, 2015.

LEE, K. N.. Compass and gyroscope: integrating science and politics for the environment. Washington: Island Press, 1993.

LEITE, M. C. F.; GASALLA, M. A.. A Method for assessing Fishers? Ecological Knowledge as a Practical Tool for Ecosystem-Based Fisheries Management: Seeking consensus in Southeastern Brazil. Fisheries Research, v.145, p.43-53, 2013. DOI: http://doi.org/10.1016/j.fishres.2013.02.013

LIMA, B. B.; VELASCO, G.. Estudo piloto sobre o autoconsumo de pescado entre pescadores artesanais do estuário da Lagoa dos Patos, RS, Brasil. Boletim do Instituto de Pesca, v.38, n.4, p.357-367, 2012. 
LUBCHENCO, J.. Entering the century of the environment: a new social contract for science. Science, v.279, n.5350, p.491-497, 1998. DOI:

http://doi.org/10.1126/science.279.5350.491

MARTINS, A. S.; PINHEIRO, H. T.; LEITE JUNIOR, N. O.. Biologia reprodutiva do camarão-sete-barbas no litoral centro-sul e sul do Espírito Santo, Brasil. Boletim do Instituto de Pesca, v.39, p.205-215, 2013.

MARTINS, A. S.; RABELO, L. B.; DOXSEY, J. R.; SOUSA, C. R.; FANCHIOTTI-MEIRELES, A.; RODRIGUES, C. M.; PIZETTA, G. T.; ARAÚJO, J. S.; SANTOS, L. B.; ZAMBON, M. C.; SILVA, M. P. C.. A pesca de camarão em Conceição da Barra, Espírito Santo, como um estudo multidisciplinar do colapso de um sistema pesqueiro. In: HAIMOVICI, M.; ANDRIGUETTO, J. M.; SUNYE, P. S.. A pesca marinha e estuarina no Brasil. Rio Grande: Furg, 2014. p.126-133.

MARTINS, A. S.; SANTOS, L. B.; SILVA, M. P. C.; DOXSEY, J. R.; SOUSA, C. R.; MEIRELES, A. F.; RODRIGUES, C. M.; PIZETTA, G. T.; ARAÚJO, J. S.; ZAMBON, M. C.; RABELO, L. B.. A rápida expansão recente da pesca de Itaipava, suas causas e consequências: um estudo de caso. In: HAIMOVICl, M.; ANDRIGUETTO, J. M.; SUNYE, P. S.. A pesca marinha e estuarina no Brasil, Rio Grande: Furg, 2014. p.134-146.

MARTINS, A. S.; SANTOS, L. B. S.; PIZETTA, G. T.; RODRIGUES, C. M.; DOXSEY, J. R.. Estudo interdisciplinar dos sistemas pesqueiros marinhos do estado do Espírito Santo, Brasil, utilizando o método RAPFISH. In: HAIMOVICI, M.. Sistemas Pesqueiros e estuarinos do Brasil. Rio Grande: FURG, 2011. p.55-65.

MARTINS, N. G.; RODRIGUES, D. A.; RIBEIRO, G. M.; FREITAS, R. R.. Avaliação da atividade pesqueira numa comunidade de pescadores artesanais no Espírito Santo, Brasil. Revista de gestão costeira integrada, v.15, n.2, p.65-275, 2015. DOI: http://doi.org/10.5894/rgci514

MCLEOD, K.; LESLIE, H.. Ecosystem based management for the oceans. Washington: Island Press, 2009.

MERÇON, F. A. M.. Entre o desenvolvimento econômico e a preservação ambiental: $O$ Caso da Pesca Artesanal e a Configuração de um Cenário de Injustiça Ambiental em Regência Augusta/ES. Dissertação (Mestrado) - Universidade Federal do Espírito Santo, Vitória, 2014.

MOURA, G. G. M.. Avanços em oceanografia humana: o socioambientalismo nas ciências do mar. São Paulo: Paco, 2017.

MOYA-ANEGÓN, F.; CHINCHILLA-RODRÍGUEZ, Z.; CORERAÁLVAREZ, E.; VARGAS-QUESADA, B.; MUÑOZ-FERNÁNDEZ, F. J.; HERRERO-SOLANA, V.; GRANADA, U.. La producción científica de la Universidad de Granada (SCI, 1991-99). Revista Española de Documentación Científica, v.28, n.2, p.170-195, 2005.

MUSIELLO-FERNANDES, J.; ZAPPES, C. A.; HOSTIM-SILVA, M.. Small-scale shrimp fisheries on the Brazilian coast: Stakeholders perceptions of the closed season and integrated management. Ocean \& Coastal Management, v.148, p.89-96, 2017. DOI: http://doi.org/10.1016/j.ocecoaman.2017.07.018(2017)
MUSIELLO-FERNANDES, J.; ZAPPES, C. A.; HOSTIM-SILVA, M. Small-scale fisheries of the Atlantic seabob shrimp (Xiphopenaeus kroyeri): Continuity of commercialization and maintenance of the local culture through making public policies on the Brazilian coast. Ocean \& Coastal Management, v.155, p.76-82, 2018. DOI: http://doi.org/10.1016/j.ocecoaman.2018.01.033

MUSIELLO-FERNANDES, J.; VIEIRA, F. V.; FLORES, R. M.; CABRAL, L.; ZAPPES, C. A.. Pesca artesanal e as interferências sobre a atividade na mesorregião central do Espírito Santo. Boletim do Museu de Biologia Mello Leitão, v.40, n.1, p.121, 2018.

MUSIELLO-FERNANDES, J.. Pesca camaroneira e conhecimento ecológico local dos pescadores artesanais de camarão na costa central do Brasil: implicações conservacionistas. Tese (Doutorado em Oceanografia Ambiental) - Universidade Federal do Espírito Santo, Vitória, 2018.

NARCHI, N. E.; CARIÑO, M.; MESA-JURADO, M. A.; ESPINOSA-TENORIO, A.; OLIVOS-ORTIZ, A.; CAPISTRÁN, M. M. E.; MORTEO, E.; OCHOA, Y.; BEITL, C. M.; DIAZ, T. E. M.; CERVANTES, O.; BRAVO, H. H. N.; SPALDING, A. K.; GRACEMCCASKEY, C. A.; CORONA, N.; MOURA, G. G. M.. EI Colaboratorio de oceanografía social: espacio plural para la conservación integral de los mares y las sociedades costeras. Ambiente \& Sociedade, v.18, p.285-301, 2019. DOI: http://doi.org/10.31840/sya.v0i18.1888

NASCIMENTO, M. S. V.; SASSI, R.. Análise da atividade pesqueira e das condições socioeconômicas dos pescadores artesanais de Cajueiro da Praia, Estado do Piauí, Brasil. Gaia Scientia, v.1, n.2, p.141-154, 2007

NUNES, A. G.. Argonautas do Mangue: uma etnografia visual dos caranguejeiros dos municípios da Grande Vitória. Dissertação (Mestrado) - Universidade Federal de Campinas, Campinas, 1998.

OLIVEIRA, B. L.. Impacto da Mitilicultura no desenvolvimento das comunidades tradicionais ao entorno das Praias da Cerca e Guaibura, Guarapari, ES. Monografia (Bacharelado em Oceanografia) - Universidade Federal do Espírito Santo, Vitória, 2004.

OLIVEIRA, P. C.; DI BENEDITTO, A. P. M.; BULHÕES, E. M. R.; ZAPPES, C. A.. Artisanal fishery versus port activity in southern Brazil. Ocean \& Coastal Management, v.129, p.4957, 2016. DOI:

https://doi.org/10.1016/j.ocecoaman.2016.05.005(2016)

OLIVEIRA, A. C. M.. Aspectos etnobiológicos e etnoecológicos na pesca artesanal em três municípios do litoral sul do Espírito Santo. Vitória: Universidade Federal do Espírito Santo, 2018.

PEDROSA, B. M. J.; LIRA, L.; MAIA, A. L. S.. Pescadores urbanos da zona costeira do Estado de Pernambuco, Brasil. Boletim do Instituto de Pesca, v.39, n.2, p.93-106, 2013.

PENA, P. L.; FREITAS, M. C. S.; CARDIM, A.. Trabalho artesanal, cadências infernais e lesões por esforços repetitivos: estudo de caso em uma comunidade de mariscadeiras na llha de Maré, Bahia. Ciências \& Saúde Coletiva, v.16, n.8, p.3383-3392, 2011. 
PEREIRA, G. R.. Produção da cultura e mediação social do ambiente nas pescas artesanais das comunidades vizinhas de Ubú e Parati no Espírito Santo. Dissertação (Mestrado) Universidade Federal do Espírito Santo, Vitória, 2014.

PINHEIRO, H. T.; JOYEUX, J.-C.. Pescarias multiespecíficas na região da foz do rio Doce, ES, Brasil: características, problemas e opções para um futuro sustentável. Brazilian Journal of Aquatic Science and Technology, v.11, p.15-23, 2007. DOI: http://doi.org/10.14210/bjast.v11n2.p15-23

PINHEIRO, H. T.; FERREIRA, A. L.; MOLINA, R. P.; PROTTI, L. M. C.; ZANARDO, S. C.; JOYEUX, J. C.; DOXSEY, J. R.. Profile of social actors as a tool the definition of marine protected areas: the case of the llha dos Franceses, southern coast of Espírito Santo, Brazil. Natureza \& Conservação, v.7, n.1, p.181-194, 2009.

PINHEIRO, F. C. F.; PINHEIRO, H. T.; TEIXEIRA, J. B.; MARTINS, A. S.; CREMER, M. J.. Opportunistic Development and Environmental Disaster Threat Franciscana Dolphins in the Southeast of Brazil. Tropical Conservation Science, v.12, p.17, 2019a. DOI: http://doi.org/10.1177/1940082919847886

PINHEIRO, H. T.; TEIXEIRA, J. B.; FRANCINI FILHO, R. B.; SOARES-GOMES, A.; FERREIRA, C. E. L.; ROCHA, L. A.. Hope and doubt for the world's marine ecosystems. Perspectives in Ecology and Conservation, v.17, p.19-25, 2019b. DOI: http://doi.org/10.1016/i.pecon.2018.11.001

PIZETTA, G. T.. Avaliação multidimensional dos sistemas pesqueiros da região sul do Espírito Santo, Brasil, e seus indicadores de sustentabilidade. Monografia (Bacharelado em Oceanografia) - Universidade Federal do Espírito Santo, Vitória, 2004.

RABELO, L. B.. A pesca do camarão em Conceição da Barra, ES, como um estudo multidisciplinar do colapso de um sistema pesqueiro. Monografia (Bacharelado em Oceanografia) - Universidade Federal do Espírito Santo, Vitória, 2006.

RAMIRES, M.; CLAUZET, M.; BARRELL, W.; ROTUNDO, M. M.; SILVANO, R. A.; BEGOSSI, A.. Fishers' knowledge about fish trophic interactions in the southeastern Brazilian coast. Journal of Ethnobiology and Ethnomedicine, v.11, n.1, p.211, 2012. DOI: http://doi.org/10.1186/s13002-015-0012-8

RIBEIRO, L. C.; SIQUEIRA, M. P.. Portos e cidades: expansão e modernização dos portos de Vitória (sec. XX-XXI). Dimensões, v.28, p.385-412, 2012.

ROCHA, K. S.; SILVA, R. V.; RANDOW, R. R.. Uma análise da percepção ambiental e transformação socioeconômica de uma comunidade de pescadores artesanais em região estuarina no sudeste do Brasil. Brazilian Journal of Production Engineering, v.11, 2012.

ROSTAING, H.. La bibliométrie et es techniques. Tolouse: Science de la Société Marselle, 1997.

SANTOS, E. C.; SAMPAIO, C. L. S.. A Pesca Artesanal na Comunidade de Fernão Velho, Maceió (Alagoas, Brasil): de Tradicional a Marginal. Revista de Gestão Costeira Integrada, v.13, n.4, p.413-424, 2013. DOI: http://doi.org/10.5894/rgci428
SANTOS, M. P. N.; SEIXAS, S. A.; MARESCHI, R. B.; HANAZAKI, N. C.; COSTA, M.; SCHIAVETTI, A.; DIAS, J. A.; AZEITEIRO, U. M.. A Pesca enquanto Atividade Humana: Pesca Artesanal e Sustentabilidade. Revista de Gestão Costeira Integrada, v.12, n.4, p.405-427, 2012.

SARTORE, M. S.; PEREIRA, S. A.; RODRIGUES, C.. Aracaju beach bars as a contested market: Conflicts and overlaps between market and nature. Ocean \& Coastal Management, v.179, p.828-837, 2019. DOI:

http://doi.org/10.1016/j.ocecoaman.2019.104828

SEVERINO A. J.. Metodologia do trabalho científico. São Paulo: Cortez, 2002.

SILVA, A. C. T.; VALENTIN, J. L.; VIANNA, M.. Competition for space between fishing and exploratory oil drilling, observed from a drilling platform in the Espírito Santo basin, southeastern Brazil. Brazilian Journal of Oceanography, v.63, n.1, p.33-41, 2015. DOI: http://doi.org/10.1590/S167987592015073306301

MARTINS, Q. S.. A relação saúde-doença entre mulheres pescadoras artesanais de São Mateus, ES. Dissertação (Mestrado) - Escola superior de ciências da Santa Casa de Misericórdia de Vitória, Vitória, 2015.

SILVA, A. L.. Entre tradições e modernidade: conhecimento ecológico local, conflitos de pesca e manejo pesqueiro no rio Negro, Brasil. Boletim do Museu Paraense Emílio Goeldi. Ciências Humanas, v.6, n.1, p.141-163, 2011. DOI: http://doi.org/10.1590/S1981-81222011000100009

SINGH, G. G.; TAM, J.; SISK, T. D.; KLAIN, S. C.; MACH, M. E.; MARTONE, R. G.; CHAN, K. M. A.. A more social science: barriers and incentives for scientists engaging in policy. Frontiers in Ecology and the Environment, v.12, n.3. p.161166, 2014. DOI: http://doi.org/10.1890/130011

STEIN, C. E.. Dinâmica da frota linheira de Itaipava, ES. Monografia (Bacharelado em Oceanografia) - Universidade Federal do Espírito Santo, Vitória, 2006.

STOJANOVIC, T. A.; FARMER, C. J. Q.. The development of world oceans \& coasts and concepts of sustainability. Marine Policy, v.42, p.157-165, 2013. DOI: http://doi.org/10.1016/j.marpol.2013.02.005

STONICH, S.; MANDELL, D.. Political ecology and sustainability science: opportunity and challenge. In: HORNBERG, A.; CRUMLEY, C.. The World System and the Earth System. Walnut Creek: Left Coast Press, 2007. p.258267.

TAVARES, M. N.. Mapeando e valorizando o conhecimento ecológico tradicional na gestão de áreas marinhas protegidas. Dissertação (Mestrado) - Universidade Nova de Lisboa, Lisboa, 2013.

TEIXEIRA, J. B.. Conhecimento ecológico tradicional como ferramenta para mapeamento de ambientes marinhos. Dissertação (Mestrado) - Universidade Federal do Espírito Santo, Vitória, 2011.

TEIXEIRA, J. B.; LIMA, A. C.; BOECHAT, F. P.; RODRIGUES, R. L.; FREITAS, R. R.. Potencialidade social e econômica da 
pesca e maricultura no Estado do Espírito Santo, Brasil.

Revista de Gestão Costeira Integrada, v.12, n.4, p. 569-575, 2012. DOI: http://doi.org/10.5894/rgci372

TEIXEIRA, J. B.; MARTINS, A. S.; PINHEIRO, H. T.; SECCHIN, N. A.; MOURA, R. L.; BASTOS, A. C.. Traditional ecological knowledge and the mapping of benthic marine habitats. Journal of Environmental Management, v.115, p.241-250, 2013. DOI: http://doi.org/10.1016/j.jenvman.2012.11.020

TIENGO, R. D. P.. Manguezais sob uma perspectiva social e econômica: percepção ambiental dos serviços ecossistêmicos presentes na ilha das caieiras, Vitória, ES. Monografia (Bacharelado em Geografia) - Universidade Federal do Espírito Santo, Vitória, 2017.

VIEIRA, L. H.. "Zonas de Sacrifício": Impactos socioambientais e a pesca artesanal em Barra do Riacho, Aracruz (ES). Revista do Programa de Pós-Graduação em Extensão Rural, v.7, n.2, p.142-168, 2018.

WEITZEL, S. R.. O papel dos repositórios institucionais e temáticos na estrutura da produção científica. Em Questão, v.12, n.1, p.51-71, 2006.
WIDMER, W. M.. A Importância da Abordagem Experimental para o Progresso da Gestão Costeira Integrada. Revista da Gestão Costeira Integrada, v.9, n.1, p.7-16, 2009. DOI: http://doi.org/10.5894/rgci142

WINIFRED, K.; TRIGUEIRO, A.; ZANETTI, D.. Os impactos socioambientais do desenvolvimento econômico na pesca artesanal: uma análise a partir dos modos de vida e de trabalho. Revista de Extensão Guará, v.2, p.25-34, 2014. DOI: http://doi.org/10.30712/guara.v2i2.9825

YOUNG, N. Y.; NGUYENB, V. M.; CORRIVEAUA, M.; COOKEB, S. J.; HINCHCET, S. G.. Knowledge users' perspectives and advice on how to improve knowledge exchange and mobilization in the case of a co-managed fishery.

Environmental Science \& Policy, v.66, p.170-178, 2016. DOI: http://doi.org/10.1016/j.envsci.2016.09.002

ZAPPES, C. A.; OLIVEIRA, P. C.; DI BENEDITTO, A. P. M. Percepção de pescadores do norte fluminense sobre a viabilidade da pesca artesanal com a implantação de megaempreendimento portuário. Boletim do Instituto de Pesca, v.42, n.1, p.73-88, 2016. DOI: http://doi.org/10.5007/1678-2305.2016v42n1p73

A CBPC - Companhia Brasileira de Produção Científica (CNPJ: 11.221.422/0001-03) detém os direitos materiais desta publicação. Os direitos referem-se à publicação do trabalho em qualquer parte do mundo, incluindo os direitos às renovaç̃oes, expansões e disseminações da contribuiç̃o, bem como outros direitos subsidiários. Todos os trabalhos publicados eletronicamente poderão posteriormente ser publicados em coletâneas impressas sob coordenação da Sustenere Publishing, da Companhia Brasileira de Produção Científica e seus parceiros autorizados. Os (as) autores (as) preservam os direitos autorais, mas não têm permissão para a publicação da contribuição em outro meio, impresso ou digital, em português ou em tradução. 